
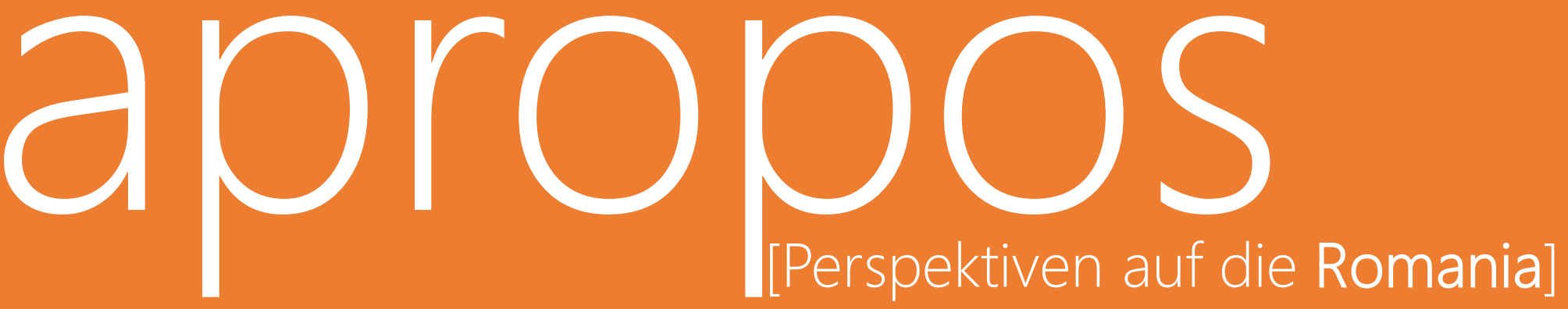

Sprache/Literatur/Kultur/Geschichte/Ideen/Politik/Gesellschaft

«Alchimie de la douleur »

Vers une compréhension systématique de la place de la douleur dans l'œuvre de Baudelaire

Pierre-Victor Haurens

apropos [Perspektiven auf die Romania] hosted by Hamburg University Press

2021, 6

pp. 251-259

ISSN: 2627-3446

Online

https://journals.sub.uni-hamburg.de/apropos/article/view/1709

Zitierweise

Haurens, Pierre-Victor. 2021. "Alchimie de la douleur'. Vers une compréhension systématique de la place de la douleur dans l'oeuvre de Baudelaire", apropos [Perspektiven auf die Romania] 6/2021, 251-259. doi: 10.15460/apropos.6.1709 
$2021, n^{\circ} 6$

pp. 251-259

doi: 10.15460/apropos.6.1709

\title{
Pierre-Victor Haurens
}

\section{"Alchimie de la douleur »}

\section{Vers une compréhension systématique de la place de la douleur dans l'œuvre de Baudelaire}

À propos de

Dotol, Giovanni. 2019. La douleur de Baudelaire, Paris : Hermann.

Dotol, Giovanni \& Mario Selvaggio (dir.) 2019. Baudelaire ou le corps de la douleur. Collection : L'Orizzonte. Paris : L'Harmattan.

Gross, Christoph. 2021. Agonie et extase. Baudelaire et l'esthétique de la douleur. Paris : Classique Garnier.

\author{
Pierre-Victor Haurens \\ est professeur agrégé et doctorant en \\ lettres modernes. \\ pvhaurens@gmail.com
}

Mots-clés

Baudelaire - douleur - esthétique - corps - théorie des affects

L'année 2021 est marquée par le bicentenaire de la naissance de Charles Baudelaire. À cette occasion, les éditeurs ont multiplié les rééditions ou les publications en hommage au poète. Les publications universitaires ne sont bien sûr pas en reste, et on a pu voir sortir un certain nombre d'ouvrages consacrés à l'auteur des Fleurs du Mal. Nous nous proposons ici d'examiner trois d'entre eux, réunis autour d'une thématique commune : la douleur. Les deux premiers ont pour caractéristique d'être centrés autour de la personne de Giovanni Dotoli, professeur émérite de Langue et Littérature françaises à I'Université de Bari, en Italie, et de Francophonie aux cours de Civilisation française de la Sorbonne. Les deux ouvrages sont parus en 2019. Le premier s'intitule sobrement La douleur de Baudelaire, paru chez Hermann, dans la collection "Vertige de la langue », fondée et dirigée par Giovanni Dotoli lui-même. II constitue un travail monographique de presque 500 pages et il est présenté par son auteur comme le résultat d'un long travail de recherche, fondé à la fois sur des thématiques de travail parallèles (ses premiers travaux universitaires sur la douleur chez Léon Bloy, cf. Dotoli 1998), et sur un goût personnel pour Charles Baudelaire. Ce mélange des dimensions caractérise I'ouvrage, nous l'aborderons plus en profondeur dans la suite de la recension. Le deuxième livre est un ouvrage collectif, Baudelaire ou le corps de la douleur, paru en juin 2019, sous la direction de Giovanni Dotoli et de Mario Selvaggio, aux éditions L'Harmattan et AGA dans la collection "L'Orizzonte », là aussi fondée et 
dirigée par Giovanni Dotoli (entre autres). L'ouvrage est la publication des actes d'un colloque qui s'est tenu à Cagliari en mars 2019, et regroupe 12 articles, des traductions en italien de poèmes de Baudelaire, ainsi qu'un certain nombre de photographies, certaines artistiques, d'autres purement illustratives, et des dessins. Le dernier ouvrage examiné ici est aussi le dernier paru. Intitulé Agonie et extase. Baudelaire et l'esthétique de la douleur, il est la version publiée de la thèse de Christoph Groß, soutenue en 2018 à l'Université libre de Berlin, sous la direction de Mme Julia Kristeva et de M. Joachim Küpper. Christoph Groß est désormais enseignant-chercheur à l'Université de Bochum. Le livre est paru chez Classiques Garnier, cette année, dans la collection «Baudelaire» qui comporte peu d'ouvrages encore mais se présente d'ores et déjà comme un apport solide et varié aux études baudelairiennes.

Comme le rappelle Giovanni Dotoli au début de son ouvrage, La douleur de Baudelaire, il est évidemment difficile de nos jours, malgré une certaine proximité de l'œuvre de Baudelaire, de trouver de nouveaux sujets d'étude sur le poète. C'est ce à quoi s'attachent en substance les deux monographies dont il est question ici. Nous laisserons un peu de côté l'ouvrage collectif, car il ne porte pas exclusivement sur la question de la douleur. Aussi surprenant que cela puisse paraître au premier abord, la douleur n'est pas une notion tellement évoquée dans les études sur Baudelaire. Une première explication réside dans sa proximité sémantique avec d'autres notions, qui ont pu prendre le pas sur celle, spécifique, de la douleur. On pense ici à la mélancolie, au spleen, à la souffrance, voire à la maladie de Baudelaire. D’autre part, la douleur a souvent été évoquée comme une thématique, ou comme un motif littéraire, et non comme un angle d'approche synthétique de l'œuvre du poète. Les deux monographies abordent donc cette question mais de manière très différente.

D’un côté Giovanni Dotoli présente sa recherche comme éminemment personnelle, et reliée à des enjeux poétiques qui lui sont propres, au-delà de la dimension universitaire de son travail. À bien des égards, son travail s'apparente plus à un essai personnel qu'à un travail universitaire classique. De fait, c'est ainsi que le présente Christoph Groß à la fin de son propre travail de recension bibliographique, dans son introduction : "Giovanni Dotoli a récemment présenté un bel essai sur La douleur de Baudelaire » (Groß 2021, 32). Nous souscrivons à son analyse quand il présente le travail de G. Dotoli comme un travail traditionnel qui lie le biographique et l'étude des textes. Sa perspective est tout autre, elle ne s'appuie pas sur la fusion voire la confusion entre l'expérience biographique et corporelle de la douleur de Baudelaire avec la place de la notion dans son œuvre et dans son système esthétique. II semble juste de dire que les deux ouvrages s'opposent radicalement dans leur style et leur méthode. L'ouvrage de G. Dotoli est plus personnel, fondé sur une expérience intime de la poésie baudelairienne, et puisant à des références diverses, " érudite[s] » (Groß 2021, 32), mais surtout concentré sur une lecture ontologique de l'œuvre : «Baudelaire est déjà pour la poésie ontologique dont parlera Stéphane Mallarmé » (Dotoli 2019, 431).

À l'opposé, C. Groß inscrit son travail dans une dimension esthétique d'abord, systématique ensuite, et suit une analyse de l'expérience esthétique qui recoupe 
les études relativement récentes sur les affects et les émotions, ainsi qu'une perspective bien plus analytique qu'ontologique des œuvres d'art, telle que la développe notamment Jean-Marie Schaeffer dans L'expérience esthétique par exemple (Schaeffer 2015), auquel C. Groß fait référence (cf. Groß 2021, 15). En caricaturant un peu, on peut dire que G. Dotoli propose une lecture plus traditionnelle, tournée vers l'ontologie de la poésie, et tirant vers le romantisme alors que $C$. Groß propose une lecture plus contemporaine, qui s'éloigne d'un dolorisme au sens biographique du terme, analysant la modernité de l'utilisation esthétique de la douleur par Baudelaire, notamment en la rapprochant des discours médicaux de l'époque. Là où G. Dotoli cherche à retrouver l'homme Baudelaire et son "corps de douleur", "Comme sa vie, l'œuvre elle-même tout entière de Baudelaire se présente comme un corps de douleur » (Dotoli 2019, 103), C. Groß étudie la poétique de Baudelaire comme « post-romantique » (Groß 2021, 396) et il est assez peu question de sa souffrance corporelle personnelle autrement que pour son rôle dans l'esthétique générale de son œuvre. De ce point de vue, ce dernier s'éloigne d'une adhésion trop proche à la figure de Baudelaire pour s'attacher à expliquer le rôle et la fonction de la douleur à la fois dans l'expérience esthétique chez Baudelaire, dans l'esthétique de sa production et enfin dans celle de sa réception. Ici, les deux titres sont explicites: le génitif lui-même un peu doloriste de La douleur de Baudelaire, chez G. Dotoli, et la mise à distance dans le sous-titre de C. Groß, Baudelaire et l'esthétique de la douleur.

\section{Un recueil disparate et inégal}

Avant d'aborder l'analyse singulière que chacune des deux monographies propose de la douleur dans l'œuvre de Baudelaire, nous allons présenter et analyser un peu plus rapidement l'ouvrage collectif et les études réunies par $G$. Dotoli et $M$. Selvaggio. En effet, ce volume ne se présente pas comme une addition très utile aux études baudelairiennes, que ce soit sur la notion de douleur ou sur d'autres thématiques. Tout d'abord, la place des articles s'arrête à la page 150. Le reste est occupé par divers travaux. Certains sont assez surprenants, il faut le dire. On trouve par exemple un «Dialogue-monologue » de G. Dotoli avec Charles Baudelaire, en vers hétérométriques. Nous laissons de côté l'évaluation de la qualité de ce poème, qui ne nous appartient pas de faire, mais on peut tout de même citer un élément de la réponse imaginée de Baudelaire à ce monologue poétique de G. Dotoli : « J'ai lui [sic] ton livre en pleurent [sic] / C'est un véritable chef-d'œuvre » (Dotoli \& Selvaggio 2019, 165). II ne nous semble pas vraiment que les actes d'un colloque soient tout à fait le lieu de publication de ce qui s'apparente à un texte personnel. Le reste du volume est d'un intérêt tout aussi discutable : on trouve des extraits de poèmes traduits en italien, et qui renvoient à une activité de lecture lors du colloque. L'importance documentaire de ces traductions est tout relatif pour un lecteur intéressé par la question de la douleur. De même pour les nombreux dessins et photographies, qui renvoient au colloque et qui ne peuvent pas véritablement intéresser le lecteur universitaire en général. On trouve notamment 20 pages de photographies purement illustratives du colloque. 
Ceci mis à part, quoique 110 pages environ sur les 266 du volume, il y a quand même un certain nombre d'articles présents. Celui de G. Dotoli, "Poète de la douleur et de la modernité » (7-24) est une reprise un peu recoupée du dernier chapitre de sa monographie "Le Corps de la douleur ». Nous l'évoquerons donc plus loin. Sur les 9 autres articles, 4 seulement s'attachent à la question de la douleur. Les autres traitent d'autres sujets concernant l'œuvre de Baudelaire: Mario Selvaggio propose une forme d'anthologie des remarques d'Yves Bonnefoy sur Baudelaire, Sonya Isaak analyse l'évocation de la peinture dans Le Spleen de Paris, Encarnación Medina Arjona examine la réception de Baudelaire à l'époque du naturalisme, en Espagne, nous passons sur les autres articles. Sans que ces textes soient dépourvus d'intérêt, ils sont souvent assez rapides, essentiellement informatifs, et ils contribuent à une forme de confusion à la lecture de l'ouvrage ne permettant pas d'approfondir une réflexion sur la question de la douleur.

En ce qui concerne les articles qui s'attachent à la notion de douleur, on trouve un texte d'Alain Rey assez narratif qui analyse quelques-unes des références artistiques et littéraires dans l'œuvre de Baudelaire, dans un rapport assez distant avec la notion de douleur; un texte de Pierre Brunel consacré au rapport entre Sainte-Beuve et Baudelaire et notamment à la lecture des Fleurs du Mal par le premier, et l'effet de tristesse qu'il y repère; un article assez confus de Paola Ricciulli, sur le titre même du recueil des Fleurs du Mal; et enfin une contribution d'Émilie Merlevede qui s'intéresse dans un article bien construit et clair à la figure du souffre-douleur dans l'œuvre de Baudelaire, article qui prend un peu de distance avec le dolorisme baudelairien et en analyse les postures et la construction. Dans l'ensemble, et pour le dire un peu vite sans doute, il s'agit d'un volume très dispensable, non seulement sur la question de la douleur, sur laquelle il n'y a pas grand-chose, mais sur Baudelaire en général, à part pour des points de détail ou un aperçu surtout informatif.

\section{Un essai impressionniste et une somme d'érudition}

Passons désormais à l'ouvrage de G. Dotoli, La douleur de Baudelaire. Ce livre, assez long puisqu'il fait presque 500 pages, constitue la somme du travail de son auteur sur l'œuvre de Baudelaire, qu'il se propose d'aborder à partir d'une notion qui lui tient à cœur et qu'il considère maltraitée, ou du moins délaissée dans l'étude de Baudelaire, celle de la douleur. Son projet est cependant un peu malaisé à cerner, notamment car il repose sur une recherche volontairement un peu vague et ontologique: "Mon projet c'est de chercher la poésie et son abîme, et son point d'origine, chez Baudelaire » (Dotoli 2019, 10). On retrouve ici des termes certes importants dans une certaine réception de la poésie, mais dont la puissance d'évocation n'a d'égale que leur grande généralité et leur flou conceptuel. De fait, l'ouvrage de G. Dotoli s'apparente à un éloge de la poésie baudelairienne, à travers un grand nombre de déclarations sans doute bien fondées, mais qui égrènent le texte comme autant de jugements gratuits et peu articulés à une démonstration bien claire. "Non, Baudelaire n'a pas voulu son chemin de douleur. C'est la vie qui l'a créé, et il s'y est bien adapté, en grand, en vrai Poète » (Dotoli 2019, 15) : cette citation montre bien qu'il s'agit autant d'une lecture de ce qu'est la poésie et la vie 
de Baudelaire par G. Dotoli qu'une analyse argumentée de la notion de douleur dans les textes du poète. De fait, l'auteur ne s'en cache pas: "Je suivrai donc le chemin de la douleur de Charles Baudelaire en critique, et surtout en poète " (idem). La perspective est honnête, mais elle rend parfois malaisée la discussion des thèses de l'auteur, car elles se situent à mi-chemin entre des analyses citées et approfondies (de ce point de vue le livre est très documenté et constitue une mine énorme de citations de l'œuvre de Baudelaire mais aussi de certaines de ses commentateurs), et des affirmations un peu péremptoires et elles-mêmes doloristes. La méthode est donc bien, comme le décrit C. Groß, proche de l'analyse biographique traditionnelle. En ce sens, à part pour un tableau synthétique des thématiques et des motifs associés à la douleur, il sera plus utile au lecteur de s'en tenir à une biographie en bonne et due forme de Baudelaire. Le deuxième chapitre est par exemple une version abrégée de la vie du poète, avec de longues citations de la correspondance, sous l'angle de la douleur. De fait l'ouvrage oscille entre la tentation d'un essai poétique libre sur Baudelaire, à l'instar des travaux d'Yves Bonnefoy (cf. Bonnefoy 2000, 2011 \& 2014), que G. Dotoli prend souvent pour modèle ( Un lecteur exceptionnel, Yves Bonnefoy, le plus grand poète français après Guillaume Apollinaire », Dotoli 2019, 14), et une étude plus classique et universitaire.

Le problème principal de l'essai, ou son avantage le plus certain, selon le point de vue avec lequel on le lit, est son aspect impressionniste. En effet, le texte de G. Dotoli se présente comme une rhapsodie de citations (de poèmes, d'extraits de la correspondance, de sources secondaires, etc.) entrecoupée de gloses assez rapides. Le texte est donc brillant pour amasser des ressources illustrant la présence de la douleur dans la vie et dans l'œuvre, mais l'argumentation elle-même fonctionne par accumulation et arguments d'autorité plutôt que par définition et analyse solide. De fait, G. Dotoli répète à plusieurs reprises que « la douleur est le chemin central de l'œuvre de Charles Baudelaire » (Dotoli 2019, 40) mais on ne sait jamais exactement pourquoi. La présence de la thématique semble la seule preuve, dont on veut bien convenir, mais cela ne nous apprend pas grand-chose sur la fonction de cette douleur et son sens. Une brève citation seulement, pour illustrer de manière symptomatique la méthode de l'auteur, fondée sur des extraits comme preuve évidente : "La correspondance va tout nous confirmer » (idem). De fait, on ne compte plus les affirmations au présent de vérité générale, illustrée par une simple citation non commentée. Le texte se présente ainsi comme un immense amoncellement de citations diverses et variées, censées illustrer l'omniprésence de la thématique dans l'œuvre et la vie de Baudelaire. En ce sens, on peut le considérer comme un excellent guide de lecture thématique dans l'œuvre du poète. Ainsi, la table des matières, constituée de trois parties, montrant d'abord la présence de la douleur chez Baudelaire (avec des entrées nominales la plupart du temps comme : le Corps, le Temps, le Sexe, l'Argent...), ensuite les moyens par lesquels Baudelaire essaie de sublimer la douleur (on retrouve là aussi des entrées nominales: la Beauté, la Femme, le Rêve...), enfin, les moyens que Baudelaire a de lutter contre la souffrance (mais cette partie ressemble beaucoup à la deuxième, brièvement intitulée " Ailleurs » avec des entrées comme le Travail, la Drogue...). On voit bien 
ainsi comment le livre procède, par sauts thématiques et avalanche de citations. La méthode a sans doute son utilité mais se rapproche du fonctionnement d'un dictionnaire ou d'une encyclopédie, plutôt que d'un essai (ce dont il se rapproche le plus) ou d'un ouvrage universitaire (ce qu'il ambitionnait peut-être d'être).

À l'inverse, il est malaisé de discuter les thèses de $\mathrm{G}$. Dotoli car il faudrait prendre chaque affirmation une à une, et l'ouvrage ne présente pas de programme général si ce n'est celui de suivre le chemin de Baudelaire. Ici apparaît tout de même une thèse, répétée plusieurs fois : "[Le poète] cherche une route de salut 》 (Dotoli $2019,335)$. Le lien en fait avec la douleur car "La douleur est 'un moyen de salut' » (Dotoli 2019, 340). Le livre se clôt d'ailleurs sur cette image: "J'espère avoir convaincu le lecteur à suivre quelques étincelles de ce même chemin de salut. » (Dotoli 2019, 439). On comprend assez bien la méthode, qui consiste à présenter Baudelaire, sa vie et son œuvre, comme un guide sur le chemin du salut. Guide qui passe par un rôle central de la douleur moyen d'accéder au salut. On voit ainsi que le dolorisme baudelairien n'est pas tant analysé qu'érigé en modèle.

L'ouvrage se termine assez significativement sur un chapitre intitulé « Le Corps de la douleur » et constitué de 16 représentations de Baudelaire, sans commentaire. Le livre contient une large bibliographie, qui, à l'instar du reste du volume, sera utile comme outil de travail. Dans l'ensemble, cet ouvrage n'aidera pas vraiment celles et ceux qui souhaitent travailler sur la notion de douleur. En revanche, les autres, intéressés par exemple par la thématique et souhaitant parcourir l'œuvre par ce biais, seront servis. Enfin, un nombre sans doute plus restreint pourra être séduit par la vision propre de G. Dotoli, mais même là, il faudra grapiller les remarques dispersées dans l'ouvrage.

\section{Une étude systématique de la douleur dans l'esthétique baudelairienne}

Le dernier ouvrage évoqué est, à notre sens, le plus riche et le plus stimulant sur cette question de la douleur dans l'œuvre de Baudelaire. La table des matières est symbolique de cette grande richesse. En effet, dans une première partie intitulée "Apex affectus », C. Groß s'attache d'abord avec une grande rigueur à mettre en perspective la notion de douleur. Cette partie est d'ambition assez historique d'une part, puisqu'elle s'appuie sur le rapport esthétique de la douleur et du beau depuis l'Antiquité jusqu'à Baudelaire, assez théorique d'autre part, puisqu'elle vise à clarifier la notion de douleur en l'inscrivant dans le champ plus large de l'étude des émotions esthétiques. II y a là un enjeu capital car cela permet de faire sortir la douleur d'une certaine passivité et de l'amener vers un enjeu plus dynamique et, à terme, créatif. De fait, une des idées majeures de l'ouvrage est ce passage du sentir au faire, qui permet de rendre compte de l'importance de la notion de douleur dans l'œuvre baudelairienne et pas seulement dans sa vie:

" l'idée d'une concession volontaire à la douleur qui transforme la passivité du souffrir en une activité créatrice en la canalisant vers un faire télique. Cette poïétique de la souffrance se présente comme purification et élévation de la vie affective du sujet souffrant à travers un approfondissement artistique de l'expérience de la douleur. » (Groß 2021, 398) 
On comprend bien ici comment la douleur devient opérante et n'est plus seulement le stigmate d'un martyr poétique. Sans éluder la question du dolorisme au sens d'un pathétisme parfois larmoyant, C. Groß prend le soin de bien en distinguer les excès du rôle esthétique de la douleur. En ce sens, il prend toujours en compte les enjeux littéraires de la notion: "Dépassant les topoï d'affect de la tradition littéraire, Baudelaire s'investit dans l'élaboration artistique d'une émotivité s'enrichissant de la douleur " (Groß 2021, 394). Dès cette première partie on comprend les enjeux du travail de $C$. Groß : tenir à distance le parallèle entre la douleur propre à la vie de Baudelaire et la douleur manifestée dans ses textes. La mise en perspective historique aide à mettre à distance ce biais, il est vrai très fréquent dans la critique baudelairienne.

Le deuxième enjeu est de s'éloigner d'un parallèle entre la douleur de la vie de Baudelaire et son supposé pessimisme. Rendre une effectivité, et par la suite un pouvoir de création à la douleur, c'est échapper à une vision trop réductrice ou vaine de la douleur. C. Groß s'inscrit ainsi dans la lignée de travaux interdisciplinaires qui, depuis quelques dizaines d'années maintenant, cherchent à conjuguer les apports des sciences dites exactes (médecine, neurosciences), avec les études dites culturelles (sociologie, anthropologie, cultural studies). On peut regrouper certaines de ces études sous l'appellation de théorie des affects. L'originalité du livre est de faire fond sur l'apport de ces études et d'en proposer une application littéraire et esthétique, sans non plus surdéterminer le rôle de facteurs sociaux-économiques dans la constitution de l'esthétique baudelairienne.

Dans une seconde partie, intitulée "Souffrir, c'est sentir », C. Groß repart du paradoxe de l'expérience esthétique désagréable pour montrer comment Baudelaire élargit et enrichit la fonction de la douleur. Le «Je » poétique baudelairien, en s'appuyant sur le nouveau lexique médical de la nervosité et celui de l'irritabilité, s'appuie sur une sensibilité moderne, fondée justement sur la douleur. Mais celleci n'est pas une soustraction à l'expérience du monde, mais au contraire un moyen de l'intensifier. En ce sens, on peut comprendre le titre du livre Agonie et extase comme l'évocation de cette douleur qui n'est pas seulement dysphorique, mais plutôt un opérateur d'intensité. Cette approche permet à C. Groß de relire certains textes à la lueur de cette notion, comme "Une mort héroïque », poème en prose du Spleen de Paris, et qui fait l'objet de toute une sous-section.

La troisième partie poursuit cette analyse de l'excitabilité nerveuse et donc de la douleur comme manière de sentir. Citons ici C. Groß qui résume les enjeux :

« Dans l'univers fictionnel de la poésie baudelairienne, la douleur devient par conséquent l'indice d'une émergence du réel et désigne de cette façon un surgissement violent du sensible qui résiste d'une part à la virtualité de l'art et suscite en même temps un désir de transcendance et de pacification. » (Groß 2021, 170-171)

Soulignons qu'une des originalités et des forces de l'ouvrage est de constamment prendre en compte la dimension historique de l'œuvre baudelairienne, en indiquant tout ce qu'elle doit au lexique médical de l'époque sur les nerfs et la nervosité, ou du moins les points de transition entre le domaine poétique et le domaine médical. 
Dans sa quatrième partie, et comme un pendant aux deux précédentes, C. Groß entame l'étude des phénomènes d'anesthésie qui concernent doublement la douleur. Sont étudiés ici les moyens par lesquels on pourrait oublier, nier, effacer la douleur, et qui sont, comme on sait, très présents chez Baudelaire sous la forme des paradis artificiels. Mais ils ne sont jamais tout à fait l'opposé de la douleur qu'un moyen de la transformer et de l'accentuer encore plus. Dans cette partie l'auteur nous propose une relecture du projet baudelairien à partir du poème « Alchimie de la douleur ». La douleur reste essentielle, comme l'état qui appelle non seulement un remède mais garantit dans le même temps une source à la créativité poétique. De fait, Baudelaire maintient en permanence une tension dans sa poétique entre ces pôles que sont l'excitation nerveuse douloureuse et son soulagement potentiel. C. Groß consacre ainsi une trentaine de pages à la démonstration de la réversibilité d'une part, et d'un processus d'alchimie de l'autre, en se fondant sur l'analyse du sonnet "Alchimie de la douleur ». Comme le dit l'auteur, ce poème permet de comprendre comment Baudelaire fait de la douleur une expérience qui n'est pas tant une destruction du projet poétique global qu'une partie intégrante de ce projet même.

Enfin, à l'aide des enjeux religieux sous-jacents dans la poétique baudelairienne, $C$. Groß fait le lien entre les deux dimensions présentes dans la perception de la douleur (I'une médicale, l'autre religieuse voire mystique). L'auteur s'y intéresse donc aux représentations religieuses bien connues dans l'œuvre de Baudelaire, mais afin d'expliquer en quoi Baudelaire est moins intéressé par un contenu doctrinal que par un vivier d'images et d'idées qui vont nourrir sa propre conception de la douleur et servir de modèle à d'autres productions esthétiques. L'auteur abord aussi dans cette dernière partie la détermination la plus approfondie de ce qu'il a appelé en introduction le "dolorisme esthétique » (Groß 2021 , 17) de Baudelaire. Reprenant l'expression de "sensualisme de la profondeur » à Barbey d'Aurevilly, l'auteur analyse le rapport au matériau religieux dans l'œuvre comme une intériorisation de la douleur, qui renonce à la transcendance de la foi pour investir la douleur dans une expérience sensible et esthétique qu'est l'art, et la poésie en particulier. C. Groß résume ainsi le parcours accompli :

«Alors, la douleur, placée dans un premier temps sous le signe d'un heurt avec le réel dont l'impact est catalysé par la surexcitabilité nerveuse-, devient dans un second temps le seuil ouvrant la voie à une intériorité qui serait susceptible de renforcer le relief émotionnel de l'expérience esthétique. Elle ne signifie plus un enfermement dans le corps, mais se transforme en signe d'une ouverture affective au dedans. » (Groß 2021, 401)

\section{De la douleur-biographie à la douleur-affect et nouvelles perspectives pour les études littéraires}

Dans l'ensemble, la lecture de ces ouvrages fait apparaître la nécessité d'interroger à nouveaux frais certains éléments de la poétique baudelairienne, en les éloignant sans doute des interprétations plus traditionnelles qui reposent sur une trop grande proximité avec le matériau biographique, désormais très bien connu. L'ouvrage de C. Groß propose ainsi un triple travail. D'abord, celui d'une mise en 
perspective historique et culturelle des enjeux d'une notion par ailleurs omniprésente mais souvent trop vite confondue avec d'autres. Ensuite, il propose un vrai travail de reconstitution de la poétologie baudelairienne, à travers une relecture minutieuse des nombreuses remarques poétiques dispersées dans l'œuvre. Cela permet de relire avec une plus grande systématicité des effets par ailleurs connus de l'esthétique de Baudelaire, mais en les expliquant plus en profondeur et en ne se méprenant pas sur le caractère secondaire de la douleur, auparavant simple motif. Enfin, son travail permet d'innerver les études baudelairiennes des apports de la recherche plus récente, notamment sur les affects, sans céder au pathétisme esthétique, et sans perdre de vue les enjeux littéraires. À cet égard, gageons que cet ouvrage saura vivifier d'autres aspects de l'étude des affects en littérature, et notamment pour le romantisme.

\section{Bibliographie}

Bonnefoy, Yves. 2000. Baudelaire: La Tentation de I'oubli. Paris: Bibliothèque nationale de France.

BonNeFoy, Yves. 2011. Sous le signe de Baudelaire. Paris : Gallimard.

BONNEFoy, Yves. 2014. Le siècle de Baudelaire. Paris : Le Seuil.

Dotolı, Giovanni. 1970. Situation des études bloyennes, suivi d'une bibliographie de 1950 à 1969. Paris : Nizet.

DоTOLI, Giovanni. 1998. Autobiographie de la douleur. Léon Bloy, écrivain et critique. Paris : Klincksieck.

SCHAEFfER, Jean-Marie. 2015. L'expérience esthétique. Paris : Gallimard. 